\title{
Effects of Postnatal Environmental Tobacco Smoke on Cardio-Respiratory Control in Newborn Lambs
}

\author{
Sally Al-Omar ${ }^{1,2,3}$, Virginie Le Rolle ${ }^{1,2}$, Nathalie Samson ${ }^{3}$, Jean-Paul Praud ${ }^{3}$, Guy Carrault ${ }^{1,2}$ \\ ${ }^{1}$ INSERM, U1099, Rennes, F-35000, France \\ ${ }^{2}$ Université de Rennes 1, LTSI, Rennes, F-35000, France \\ ${ }^{3}$ Université de Sherbrooke, Canada
}

\begin{abstract}
While prenatal environmental tobacco smoke (ETS) exposure has been shown to be a major cause of sudden infant death syndrome, the effects of postnatal ETS exposure have been less studied. The objective of this study is to explore the effects of 15-days postnatal exposure to 20 cigarettes/day on heart rate variability (HRV), respiratory rate variability (RRV) and cardiorespiratory interrelations in newborn lambs. On postnatal day 16, sixhours polysomnographic recording was obtained from six control lambs exposed to room air (group $\mathrm{CO}$ ) and six lambs exposed to 20 cigarettes/day (group C20) for the first 15 days of life. ECG and respiratory movements (RSP) were continuously monitored. Several analyses were performed on these signals, including heart rate variability analyses (on the RR intervals extracted from the ECG), respiratory rate variability analyses (on different time series extracted from the breathing signal) and cardiorespiratory interrelations analyses (on both the $R R$ interval and the breathing signal). The results suggest that ETS exposure for the first 15 postnatal days leads to slight alterations of cardiorespiratory control, which predominate on respiration.
\end{abstract}

\section{Introduction}

The early postnatal period is critical for the newborn, due to the immaturity of many vital functions such as breathing and cardiac function. In fact, the health problems of newborns affecting the autonomic nervous system that controls the heart and breathing can have dramatic consequences such as apneas, bradycardias and sudden infant death syndrome (SIDS) [1].

Following the worldwide campaign to promote supine sleep in infants, perinatal environmental tobacco smoke (ETS) seems to be the most important preventable risk for SIDS [2]. In fact, prenatal and postnatal ETS exposure increases SIDS risk from 2 to 5 times [3]. However, most studies have considered the effects of nicotine alone instead of ETS exposure. Nicotine was found to be the major factor in cigarette smoke that acts as a developing neurotoxicant and is responsible for the main harmful effects [4]. Results showed that prenatal nicotine exposure causes alterations of lung and central nervous system function [5]. To our knowledge, however, studies of the effects of postnatal ETS exposure on cardiorespiratory control are rare. The aims of our study were to assess the effect of postnatal ETS exposure on the autonomic nervous system (ANS) by analyzing heart rate variability (HRV), respiratory rate variability (RRV) and cardiorespiratory interrelations, by applying several interrelations methods.

The paper is divided into three main sections. In the first one, we describe the experimental protocol and we present the different methods used to analyze the HRV, the RRV and the cardiorespiratory interrelations. In the second section, we introduce the results of the analyses and we discuss them in the final section.

\section{Materials and methods}

\subsection{Experimental protocol}

Experiments were performed on 12 lambs born at term. The protocol was approved by the Committee for Animal Care and Experimentation of the University of Sherbrooke, Canada. Surgery was performed two days before the experiment under general anesthesia following the procedure detailed in [1] for catheter implantation. Respiratory thoraco-abdominal movements (RSP) were monitored with respiratory inductance plethysmography (Respitrace; NIMS Inc., Miami, FL, USA). Two electrocardiogram (ECG) channels were acquired using a couple of ECG100 modules (Biopac Systems, Inc. Santa Barbara, CA, USA). All signals were sampled at $1000 \mathrm{~Hz}$ and recorded on a PC, using the MP100A data acquisition system and Acknowledge 3.7.3 software (Biopac Systems Inc. Santa Barbara, CA, USA).

The twelve lambs were randomly divided into two groups: 6 control lambs exposed to room air (group $\mathrm{C} 0$ ) and 6 lambs exposed to 20 cigarettes/day (group C20) for the 
first 15 days of life. The exposure duration was 8 hours per day divided into two periods of 4 hours separated by a 30 min pause at noon for bottle-feeding. An automatic custom-built cigarette-smoking machine was used, with a preset configuration of 2s puff duration, $35 \mathrm{ml}$ puff volume, according to ISO 3308 norms, with a 30 s interval between 2 puffs [6]. After 15 days of ETS exposure, a 6 hours polysomnographic recording was performed in each lamb. Lambs were euthanized at the end of the recording.

\subsection{Data processing}

$\mathrm{RR}$ interval series were derived beat-by-beat from the ECG, manually edited, interpolated and resampled evenly at $4 \mathrm{~Hz}$. The RSP signal was also resampled at $4 \mathrm{~Hz}$ (Figure 1). The RR and RSP signals were detrended and normalized. Three time series were extracted from the RSP signal: inspiratory time (Ti), expiratory time (Te) and total breathing cycle time (Ttot) [7]. The first 5 stationary segments from the recordings were manually selected. The duration of each selected segment was 2 min.

\subsubsection{Heart rate variability}

Heart rate variability (HRV) was used to characterize heart rate modulation by the autonomic nervous system (ANS) and the balance between the sympathetic and parasympathetic systems [8]. Both linear (time and frequency domain) and nonlinear analyses were compared to evaluate the effect of postnatal ETS exposure on the HRV.

\subsubsection{Time domain parameters}

According to the Task Force on HRV[9], the following time domain variables were computed : the mean of the RR intervals and the standard deviation (SDNN), which is a measure of the global variability, and the square root of the mean squared differences of successive RR intervals (RMSSD) that reflects parasympathetic control of sinus rhythm. In addition, the skewness and kurtosis of the RR distribution were calculated.

\subsubsection{Frequency domain parameters}

The power spectrum of the RR series was computed by Welch algorithm and integrated in the low frequency band, from $0.02 \mathrm{~Hz}$ to $0.25 \mathrm{~Hz}$, and in the high frequency band, from $0.25 \mathrm{~Hz}$ to $2 \mathrm{~Hz}$ [10] to get respectively two measures: the LF power, which is the reflection of both vagal and sympathetic control of sinus node, and the HF power, which is the reflection of cardiac vagal control. These frequency bands have been previously used for newborn lambs [10]. The normalized LFnu and HFnu indices were computed as $\mathrm{LF} /(\mathrm{HF}+\mathrm{LF})$ and $\mathrm{HF} /(\mathrm{HF}+\mathrm{LF})$ respectively

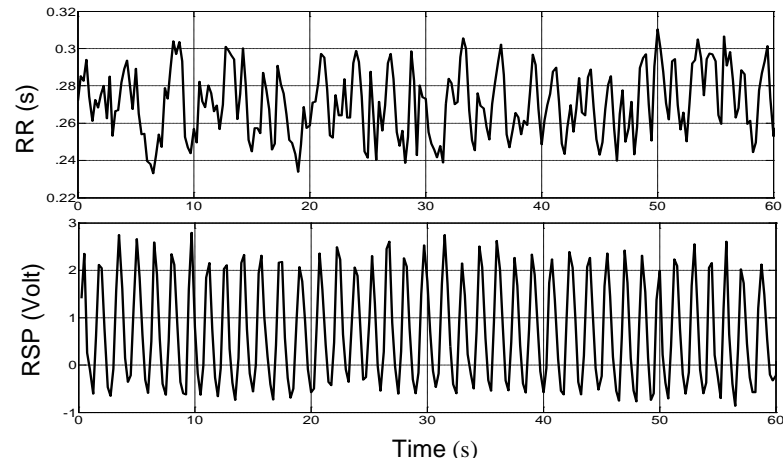

Figure 1: Example of RR signal (top) and RSP signal (bottom)

[11]. Also, the LF/HF ratio, which reflects the global cardiac sympathovagal balance (e.g., decreased LF/HF reflects more vagal and/or less sympathetic cardiac control) [10], was computed. Finally, we extracted the total power spectrum (TP) which reflects the overall autonomic activity.

\subsubsection{Nonlinear analyses}

The nonlinear methods provide an assessment of the complexity of the HRV dynamics. SD1 and SD2 parameters were extracted from the Poincaré plots, as indices of short-term variability and both short- and longterm variability respectively. Also, we calculated the sample entropy (SampEn) of the RR series as a measure of their complexity. The sample entropy is linked to the probability that two series similar for $\mathrm{N}$ points, remain similar at the next point. Lower values of entropy reflect higher regularity [10].

\subsubsection{Respiratory Variability}

Several parameters were calculated on the time series extracted from the RSP signal (Ti, Te and Ttot): the mean, the standard deviation, the skewness and the kurtosis. Similarly to the RR series, nonlinear measures were also calculated, including SD1, SD2 and SampEn.

\subsubsection{Cardiorespiratory Interrelations}

In order to explore the changes that may occur to the cardiorespiratory interrelations after 15 days of ETS exposure, we applied several methods such as: classic linear and nonlinear regression based methods, a method to evaluate the phase synchronization between the RR and the RSP series, in addition to an intuitive method based on counting the occurrence of heart beats in an inspiration and in an expiration. 


\subsubsection{Linear and nonlinear regression based methods}

The Pearson correlation coefficient $\left(\mathrm{r}^{2}\right)$ is a measure of the strength and direction of a linear relationship between RR and RSP:

$$
\mathrm{r}_{1,2}^{2}=\frac{\mathrm{E}\{(\mathrm{RR}-\mathrm{E}(\mathrm{RR}))(\mathrm{RSP}-\mathrm{E}(\mathrm{RSP}))\}}{[\operatorname{var}(\mathrm{RR}) * \operatorname{var}(\mathrm{RSP})] 1 / 2}
$$

where var(.) and $\mathrm{E}($.$) are respectively the variance and the$ expectation. The process RSP can be delayed with a timelag $(\tau)$ and the maximum of $r_{1,2}^{2}(\tau)$ is considered $r^{2}$ [12]. The non-linear correlation $\left(\mathrm{h}^{2}\right)$ is a measure of the statistical dependence between RR and RSP:

$$
\mathrm{h}_{\text {RR-RSP }}^{2}=\frac{\operatorname{var}(\mathrm{RSP})-\|\mathrm{RSP}-\mathrm{f}(\mathrm{RR})\| 2}{\operatorname{var}(\mathrm{RSP})}
$$

where $f$ is a nonlinear regression function permitting the measurement of the more or less linear similarity between $\mathrm{RR}$ and RSP [12]. The nonlinear regression coefficient was assessed in both directions, i.e., first $h^{2}{ }_{\text {RR-RSP }}\left(h^{2}\right.$ RRonRespi) and secondly $\mathrm{h}^{2}{ }_{\mathrm{RSP}-\mathrm{RR}}\left(\mathrm{h}^{2}\right.$ RespiOnRR). In both cases, one of the processes was delayed with a timelag $(\tau), h^{2}(\tau)$ was computed and the maximum $h^{2}(\tau)$ was considered $\mathrm{h}^{2}$.

\subsubsection{Phase synchronization index}

The concept of phase synchronization was previously applied to biological time series such as heart beat and respiratory rate [13], EEG[14] and EMG [15]. In order to quantify the phase synchronization between the RR and RSP series, we used an index based on the distribution of the phases $\varnothing_{\mathrm{RR}}$ and $\varnothing_{\mathrm{RSP}}$ of RR and RSP respectively:

$$
\gamma_{\mathrm{RR}, \mathrm{RSP}}=\sqrt{<\cos \emptyset_{\mathrm{RR}, \mathrm{RSP}}>^{2}+<\sin \emptyset_{\mathrm{RR}, \mathrm{RSP}}>^{2}}
$$

where $\varnothing_{\mathrm{RR}, \mathrm{RSP}}=\varnothing_{\mathrm{RR}}-\varnothing_{\mathrm{RSP}}$ and 〈.〉 denotes the average over time. The measure of synchronization strength evolves from 0 (no synchronization) to 1 (perfect synchronization) [15].

\subsubsection{RR Count during inspiration and expiration}

The mean number of heart beats that occurred in each inspiration (MeanRRInspi) was calculated, on all 2-min segments. In the same manner, we computed the same measure during expiration time and defined theMeanRRExpi.

\subsubsection{Statistical analyses}

All results are expressed as mean \pm standard deviation (SD) and rounded. To compare the results of the two groups, the non-parametric Mann-Whitney rank sum test was applied. Since the number of studied lambs was relatively small (due to the complexity of the ovine model and to ethical constraints), it was decided to discuss the presence of a significant trend, defined as $\mathrm{p}<0.1$.

\section{Results}

Table 1 details the results of the analyses explained in the previous section. Not all insignificant results of: HRV, RRV for $\mathrm{Ti}$ series, and cardiorespiratory interrelations figure in the table. For HRV, only skewness showed a difference between the two groups. For RRV, the SDNN and the SD2 of Te, the SDNN and the skewness of Ttot showed a significant change in the C20 group. Finally, $\mathrm{h}^{2}$ RSP-RR tended also to be significantly different between

\begin{tabular}{|c|c|c|c|}
\hline \multicolumn{4}{|c|}{ Heart Rate Variability Analysis } \\
\hline $\begin{array}{l}\text { Linear Analysis } \\
\text { Time Domain }\end{array}$ & CO & C20 & $\begin{array}{c}p- \\
\text { values }\end{array}$ \\
\hline Mean (ms) & $335 \pm 69.6$ & $317 \pm 41.3$ & 0.49 \\
\hline RMSSD (ms) & $9.2 \pm 9.9$ & $7.5 \pm 9.5$ & 0.35 \\
\hline Skewness & $0.5 \pm 1.4$ & $-0.01 \pm 0.6$ & 0.05* \\
\hline \multicolumn{4}{|l|}{ Frequency Domain } \\
\hline $\mathrm{LF}\left(\mathrm{ms}^{2}\right)$ & $39.1 \pm 35.1$ & $35.7 \pm 22.1$ & 0.94 \\
\hline $\mathrm{HF}\left(\mathrm{ms}^{2}\right)$ & $55.5 \pm 91.6$ & $28.2 \pm 25.3$ & 0.46 \\
\hline \multicolumn{4}{|l|}{ Nonlinear Analysis } \\
\hline SD1 & $8.5 \pm 7.6$ & $6.7 \pm 4.2$ & 0.97 \\
\hline SD2 & $15.7 \pm 6.4$ & $15.1 \pm 4.8$ & 0.99 \\
\hline \multicolumn{4}{|c|}{ Respiratory Rate Variability Analysis } \\
\hline Te & CO & C20 & $\begin{array}{c}p- \\
\text { values }\end{array}$ \\
\hline Mean (ms) & $1.1 \pm 0.2$ & $1.1 \pm 0.2$ & 0.98 \\
\hline SDNN (ms) & $0.1 \pm 0.04$ & $0.2 \pm 0.1$ & $0.05^{*}$ \\
\hline Skewness & $-0.2 \pm 0.8$ & $-0.3 \pm 1.5$ & 0.79 \\
\hline Kurtosis & $4 \pm 2.3$ & $5.9 \pm 6.2$ & 0.53 \\
\hline SD1 & $0.1 \pm 0.04$ & $0.1 \pm 0.1$ & 0.16 \\
\hline SD2 & $0.1 \pm 0.05$ & $0.2 \pm 0.1$ & $0.09 *$ \\
\hline SampEN & $2 \pm 0.2$ & $2 \pm 0.3$ & 0.34 \\
\hline \multicolumn{4}{|l|}{ Ttot } \\
\hline Mean (ms) & $1.7 \pm 0.4$ & $1.8 \pm 0.3$ & 0.4 \\
\hline SDNN (ms) & $0.1 \pm 0.05$ & $0.2 \pm 0.1$ & $0.06^{*}$ \\
\hline Skewness & $0.4 \pm 1$ & $-0.05 \pm 1.3$ & $0.09 *$ \\
\hline Kurtosis & $5.5 \pm 2$ & $5.1 \pm 4.1$ & 0.2 \\
\hline \multicolumn{4}{|c|}{ Cardiorespiratory Interrelations Analysis } \\
\hline Variables & CO & C20 & $\begin{array}{c}P \\
\text { values }\end{array}$ \\
\hline $\mathrm{R}^{2}$ & $0.08 \pm 0.1$ & $0.1 \pm 0.1$ & 0.22 \\
\hline $\mathrm{h}^{2} \mathrm{RSP}-\mathrm{RR}$ & $0.12 \pm 0.08$ & $0.16 \pm 0.09$ & $0.06 *$ \\
\hline MeanRRExpi & $2 \pm 0.5$ & $2.1 \pm 0.6$ & 0.13 \\
\hline
\end{tabular}
the two groups.

Table 1: Results of the different analyses

\section{Discussion}

Our results suggest that ETS exposure for the first 15 postnatal days leads to slight alterations of 
cardiorespiratory control, which predominate on respiration. The present study complements a previous one performed on the same database that showed that ETS exposure for the first 15 postnatal days did not have any influence on lambs' respiratory and heart rates [6].

The effects of tobacco exposure on neonatal cardiorespiratory function have been previously evaluated in newborn rats and lambs [16] [17]. Although alterations in cardiorespiratory function were found, most of the studies used levels of nicotine exposure ( $>1 \mathrm{mg} / \mathrm{kg} / \mathrm{day})$, which are much higher than the ones measured during human newborn exposure [18]. On the contrary, the lower cotinine levels measured in the urine of the lambs in the present study ensured that our postnatal ETS mimicked the conditions encountered for a newborn infant [1]. Our results further suggest and confirm that the effect of the ETS exposure on cardiorespiratory control is dosedependent [5], and concur with the view that prenatal ETS exposure appears more harmful than postnatal exposure [4].

One limitation of the present study may be that the segment duration (2 min) used for the analyses is too short. Hence, in order to further confirm these preliminary results, the same analyses will be repeated on longer segments. In addition, our studies will be extended by analyzing the two cardiorespiratory interrelations previously reported in the literature, namely the respiratory sinus arrhythmia (RSA) and the cardioventilatory coupling (CVC) [19].

\section{Acknowledgements}

Supported by the Canada Research Chair in Neonatal Respiratory Physiology allocated to JP Praud and by the French Brittany council

\section{References}

[1] Duvareille C, St-Hilaire M, Samson N, Bakirtzian P, Brisebois S, Boheimier $\mathrm{M}$, et al. Effects of postnatal environmental tobacco smoke on non-nutritive swallowingbreathing coordination in newborn lambs. Respir Physiol Neurobiol. 2013 Jan 15;185(2):446-53.

[2] Adgent MA. Environmental tobacco smoke and sudden infant death syndrome: a review. Birth Defects Res B Dev Reprod Toxicol. 2006 Feb;77(1):69-85.

[3] Liebrechts-Akkerman G, Lao O, Liu F, Sleuwen BE van, Engelberts AC, L'Hoir MP, et al. Postnatal parental smoking: an important risk factor for SIDS. Eur J Pediatr. 2011 Mar 15;170(10):1281-91.

[4] Slotkin TA. Cholinergic systems in brain development and disruption by neurotoxicants: nicotine, environmental tobacco smoke, organophosphates. Toxicol Appl Pharmacol. 2004 Jul 15;198(2):132-51.

[5] Hafström O, Milerad J, Sandberg KL, Sundell HW. Cardiorespiratory effects of nicotine exposure during development. Respir Physiol Neurobiol. 2005 Nov 15;149(1-3):325-41.

[6] Duvareille C, Beaudry B, St-Hilaire M, Boheimier M,
Brunel C, Micheau P, et al. Validation of a new automatic smoking machine to study the effects of cigarette smoke in newborn lambs. Lab Anim. 2010 Oct;44(4):290-7.

[7] Navarro X, Poree F, Beuchee A, Carrault G. Respiration signal as a promising diagnostic tool for late onset sepsis in premature newborns. Computing in Cardiology 2010;37: 947-50.

[8] Bailón R, Mainardi L, Orini M, Sörnmo L, Laguna P. Analysis of heart rate variability during exercise stress testing using respiratory information. Biomed Signal Process Control. 2010 Oct;5(4):299-310.

[9] Heart rate variability: standards of measurement, physiological interpretation and clinical use. Task Force of the European Society of Cardiology and the North American Society of Pacing and Electrophysiology. Circulation. 1996 Mar 1;93(5):1043-65.

[10] Beuchée A, Hernández AI, Duvareille C, Daniel D, Samson $\mathrm{N}$, Pladys $\mathrm{P}$, et al. Influence of Hypoxia and Hypercapnia on Sleep State-Dependent Heart Rate Variability Behavior in Newborn Lambs. Sleep. 2012 Nov 1;35(11):1541-9.

[11] Khodor N, Matelot D, Carrault G, Amoud H, Khalil M, Ville N, et al. Kernel based support vector machine for the early detection of syncope during head-up tilt test. Physiol Meas. 2014 Oct;35(10):2119-34.

[12] Loforte R, Carrault G, Mainardi L, Beuche A. Heart rate and respiration relationships as a diagnostic tool for late onset sepsis in sick preterm infants. In: Computers in Cardiology, 2006. 2006. p. 737-40.

[13] Schäfer C, Rosenblum MG, Abel HH, Kurths J. Synchronization in the human cardiorespiratory system. Phys Rev E Stat Phys Plasmas Fluids Relat Interdiscip Top. 1999 Jul;60(1):857-70.

[14] Mormann F, Lehnertz K, David P, Elger CE. Mean Phase Coherence As a Measure for Phase Synchronization and Its Application to the EEG of Epilepsy Patients. Phys. 2000 Oct;144(3-4):358-69.

[15] Hassan M, Terrien J, Karlsson B, Marque C. Interactions between Uterine EMG at Different Sites Investigated Using Wavelet Analysis: Comparison of Pregnancy and Labor Contractions. Eurasip J Adv Signal Process. 2010;2010(1):918012.

[16] Hafström O, Milerad J, Sundell HW. Altered breathing pattern after prenatal nicotine exposure in the young lamb. Am J Respir Crit Care Med. 2002 Jul 1;166(1):92-7.

[17] Bamford OS, Schuen JN, Carroll JL. Effect of nicotine exposure on postnatal ventilatory responses to hypoxia and hypercapnia. Respir Physiol. 1996 Oct;106(1):1-11.

[18] Hussein J, Farkas S, MacKinnon Y, Ariano RE, Sitar DS, Hasan SU. Nicotine dose-concentration relationship and pregnancy outcomes in rat: Biologic plausibility and implications for future research. Toxicol Appl Pharmacol. 2007 Jan 1;218(1):1-10.

[19] Galletly DC, Larsen PD. Relationship between cardioventilatory coupling and respiratory sinus arrhythmia. Br J Anaesth. 1998 Feb;80(2):164-8.

Address for correspondence.

LE ROLLE Virginie

LTSI, Beaulieu Campus, Université de Rennes 1, 35042 Rennes, France

Virginie.lerolle@univ-rennes1.fr 